Revue d'histoire de l'Amérique française

Q4. REVUE D'HISTOIRE DE L'AMÉRIQUE FRANÇAISE

\title{
Les routes à Sillery sous le régime français
}

\section{François Cantara}

Volume 49, numéro 4, printemps 1996

URI : https://id.erudit.org/iderudit/305464ar

DOI : https://doi.org/10.7202/305464ar

Aller au sommaire du numéro

\section{Éditeur(s)}

Institut d'histoire de l'Amérique française

\section{ISSN}

0035-2357 (imprimé)

1492-1383 (numérique)

Découvrir la revue

\section{Citer cet article}

Cantara, F. (1996). Les routes à Sillery sous le régime français. Revue d'histoire de l'Amérique française, 49(4), 551-566. https://doi.org/10.7202/305464ar

\section{Résumé de l'article}

À Sillery, sous le Régime français, le terme " route » ne désigne pas un chemin praticable, mais une simple ligne d'arpentage servant à borner les terres d'une même côte. Et, en raison de cette fonction, ce terme connaîtra un glissement de sens qui l'identifiera tout à fait à la côte dont il partage déjà le nom. C'est ce qu'attestent des mentions de résidence d'individus ou d'emplacement de terres, contenues dans un certain nombre d'actes notariés : on pourra se dire " de la route " aussi bien que " de la côte Saint-Ignace ", par exemple. Le " réseau routier » de Sillery, aux composantes parallèles entre elles, ne doit donc pas être confondu avec son réseau de chemins praticables qui sillonnent la seigneurie selon un tout autre tracé. 


\section{LES ROUTES À SILLERY SOUS LE RÉGIME FRANÇAIS}

FRANÇOIS CANTARA

Département d'histoire

Université de Montréal

\section{RÉSUMÉ}

À Sillery, sous le Régime français, le terme «route» ne désigne pas un chemin praticable, mais une simple ligne d'arpentage servant à borner les terres d'une même côte. Et, en raison de cette fonction, ce terme connaîtra un glissement de sens qui l'identifiera tout à fait à la côte dont il partage déjà le nom. C'est ce qu'attestent des mentions de résidence d'individus ou d'emplacement de terres, contenues dans un certain nombre d'actes notariés: on pourra se dire «de la route» aussi bien que «de la côte Saint-Ignace», par exemple. Le «réseau routier» de Sillery, aux composantes parallèles entre elles, ne doit donc pas être confondu avec son réseau de chemins praticables qui sillonnent la seigneurie selon un tout autre tracé.

\section{ABSTRACT}

In notarial documents concerning the seigneury of Sillery during the French regime, the word "route» refered to a survey line along the frontage of plots along the same cote or range rather than a roadway. The meaning of the word evolved from being a survey line to being synonymous with cote and people declared their residence indifferently as the route Saint-Ignace or the cote Saint-Ignace, for example. Thus route lines should not be confused with roadways which followed a different lay out.

C'est en voulant étudier les migrations dans la seigneurie de Sillery que nous avons été amené à en reconstituer le terrier pour toute la période du Régime français. Marcel Trudel s'était déjà attaqué à cette tâche, comme chacun sait, dans son Terrier de $1663^{1}$, en s'aidant principalement de l'aveu et dénombrement que les Jésuites produisirent, en 1678, comme tuteurs et administrateurs des Amérindiens, véritables propriétaires de cette seigneurie jusqu'en $1699^{2}$. Lucien

1. M. Trudel, Le terrier du Saint-Laurent en 1663 (1973).

2. Le 23 octobre 1699, le gouverneur Louis-Hector de Callières transfère les titres de propriété de la seigneurie de Sillery, abandonnée par les Amérindiens depuis 1687 environ, au profit des Jésuites; ANQQ, Fonds Biens des Jésuites, boîte E21/187. 
Campeau, dans un article paru en 1993, tentait de clarifier certains points concernant la topographie de Sillery et les modalités de concession de ses premières terres ${ }^{3}$. Nos propres recherches nous ont conduit à pousser encore plus loin ces premières tentatives de reconstitution de la genèse de Sillery.

Messieurs Trudel et Campeau ont mené leurs travaux sur Sillery en s'appuyant sur une documentation relativement complète. Pour notre part, le fonds «Biens des Jésuites» conservé aux Archives nationales du Québec, à Québec, nous a donné accès à un riche corpus de documents: la quasi-totalité des actes de concession des terres de Sillery, rédigés sous seing privé, ainsi que différents factum concernant les affaires courantes de la seigneurie et émanant de la main même des Jésuites, etc. Au service des archives de l'arpentage, du ministère des Ressources naturelles du Québec, nous avons pu consulter différents plans parcellaires levés par le père Druillettes, dans les années 1660 , et de nombreux procès-verbaux d'arpentage ultérieurs; mais ce qui a par-dessus tout retenu notre attention en même temps que suscité notre embarras, c'est un précieux plan cadastral de 1754, levé par l'arpenteur Ignace Plamondon, et accompagné de son procès-verbal.

Embarrassant, disions-nous, car la description que ce plan nous donne de Sillery ne concorde pas avec celle que nous offre les plans parcellaires du Père Druillettes. Cela est sans doute normal, trouverat-on, puisque près de quatre-vingts années les séparent. Certes. Mais l'embarras, loin de se dissiper, s'accroît à la lecture des nombreux actes qui ont été rédigés sur toute la période du Régime français et qui nous offrent des indications précises sur les lieux de résidence des individus en même temps que sur la localisation de leur terre: routes et chemins, à Sillery, font problème.

Prenons, pour commencer, le plus complet des trois plans parcellaires de Druillettes, dressé autour de $1665^{4}$. À cette date, la plus grande partie du territoire de Sillery est déjà répartie en trois rangées de concessions que ce plan n'identifie cependant pas nommément: ce sont, pour nous, la côte Saint-François, bornée, dans son front, par le fleuve Saint-Laurent et derrière, à la suite l'une de l'autre, les côtes de Saint-Ignace et de Saint-Michel ${ }^{5}$. Sur ce même plan, trois routes sont

3. L. Campeau, «Le Fief des Sauvages et l'organisation de Québec», Les Cahiers des Dix, 48 (1993): 9-44.

4. Pour un meilleure compréhension de ce qui va suivre, nous avons reproduit une carte de Sillery contenant les éléments les plus importants tirés des plans de Druillettes et de Plamondon.

5. Seule n'apparaît pas sur cette carte la future côte Saint-Pierre qui ne fera l'objet de concessions que dans les années 1670 . 


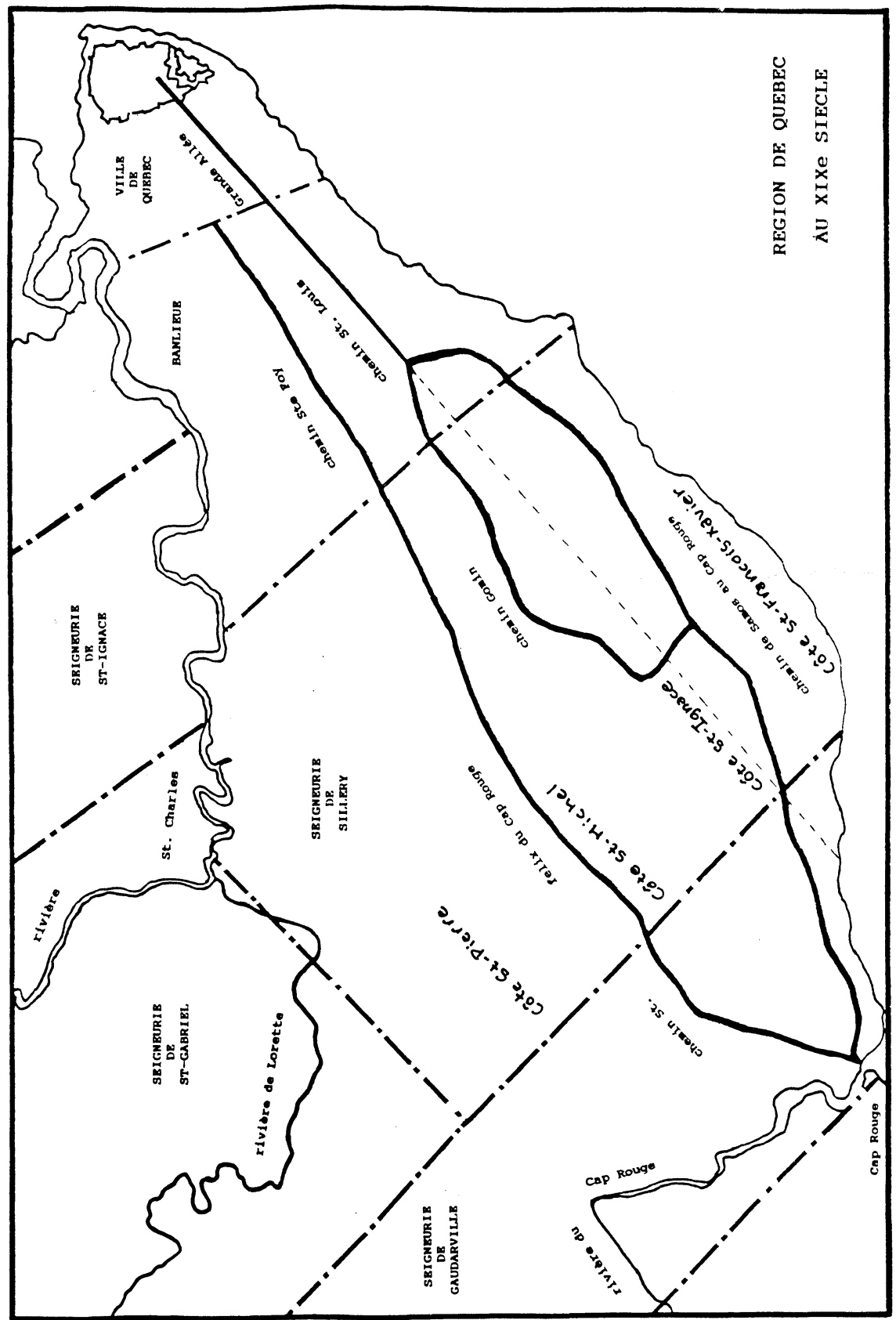


toutefois nommément identifiées: il s'agit de la «Grande Route de Kebec au Cap Rouge» et des «Route S. ignace» et «Route Sainct michel». En dehors de ces dernières, aucun chemin n'est représenté sur ce plan.

Passons maintenant au plan cadastral de Plamondon, établi en 1754. Comme on pouvait s'y attendre, quatre rangées de concessions, parallèles entre elles, se partagent dorénavant le territoire de la seigneurie: les côtes Saint-François, Saint-Ignace, Saint-Michel et Saint-Pierre. Dans la profondeur des trois premières côtes, Plamondon n'identifie que trois des quatre routes connues alors: "grande allée», »St ignace» et «St michel ${ }^{6} »$. Enfin, quatre chemins traversant le territoire de Sillery sont nommément identifiés: «le chemain qui conduit a quebec», «le chemain qui conduit de quebec a st augustin», «le chemain qui conduit de Ste foi a lorette» et, enfin, «le chemain qui conduit de lorette a quebec».

Ce qu'il y a d'étonnant quand on compare ces deux plans, c'est, premièrement, la distinction graphique que l'arpenteur de 1754 semble faire entre chemins et routes; celles-ci, rectilignes, sont représentées par des droites; ceux-là, d'allure plus ou moins régulière, sont représentés par deux lignes rompues parallèles. Deuxièment, les chemins ne passent pas là où l'on s'y attendrait: le «chemain qui conduit a quebec» et «le chemain qui conduit de quebec a st augustin» traversent respectivement le milieu des terres des côtes de Saint-François et de Saint-Michel. En fait, ils ne passent pas par un bout ou par l'autre de ces deux côtes, comme nous y avaient habitués les plans des Jésuites des années 1660 .

Une première explication qui vient à l'esprit serait que ces routes représentées sur les plans des Jésuites, et qui ont existé jadis, sont disparues à la fin du Régime français. Abandonnées par leurs utilisateurs, elles auraient été remplacées, sous une autre appellation, par les chemins représentés en $1754^{7}$.

6. Et encore le fait-il de façon erronée: si la Grande-Allée est bien à sa place sur son plan, il n'en va pas de même pour les routes Saint-Ignace et Saint-Michel qu'il situe dans la profondeur des côtes du même nom, alors qu'elles devraient en occuper le front. Son erreur vient de ce que les routes Saint-Ignace et Grande-Allée ont fini par se confondre, au milieu du XVIII siècle, lorsqu'on décida, à la suite d'une situation qui perdurait depuis le $\mathrm{XVII}^{\mathrm{e}}$ siècle, de rallonger les terres de la côte Saint-Ignace jusqu'à la Grande-Allée, au détriment de la profondeur des terres de la côte Saint-François. On s'explique mieux alors pourquoi il n'arrive pas à situer la «route St-Pierre» dont la place est déjà occupée par la «route St-Michel».

7. C'est ainsi que l'ancienne «Grande Route de Kebec au cap Rouge», des plans des Jésuites, pourrait avoir été abandonnée au profit d'un nouveau tracé, celui du «chemain qui conduit à Quebec», ce dont rendrait compte la carte de Plamondon, en 1754. 
Cette explication pourrait nous satisfaire si nous avions comme seules sources les quatre plans parcellaires des Jésuites et la carte de Plamondon. Mais il y a de nombreux autres documents (actes de concession, procès-verbaux d'arpentage, etc.), répartis sur toute la durée du Régime français, et où il n'est jamais fait mention de ces routes comme d'anciens chemins. Bien au contraire, elles sont mentionnées, sans discontinuité dans le temps, comme de véritables entités contemporaines ${ }^{8}$.

Nous avons donc cherché ailleurs une explication qui prendrait en compte cette objection et, après un examen attentif des documents, nous en sommes arrivé à cette étonnante constatation: au début de son utilisation, le terme «route», tiré probablement du langage maritime, a servi à désigner une simple ligne d'arpentage réalisée à l'aide d'une boussole ${ }^{9}$. A cette fonction de bornage des terres, on a songé, à un certain moment, à y adjoindre celle de chemin praticable. La confusion entre ces deux termes, modernes pour nous, viendrait donc de leur apparente synonymie accentuée par le fait qu'ils ont pu désigner, pendant une brève période de temps, une même réalité, c'est-à-dire une voie praticable.

Bien plus, cette ligne d'arpentage, du même nom que la côte à laquelle elle était associée, a pu désigner, dans un certain nombre d'actes notariés, le lieu de résidence des individus ou l'emplacement de leur terre: c'est ainsi que l'on a pu se dire de la route comme de la côte Saint-Ignace, Saint-François, Saint-Michel ou Saint-Pierre.

\section{LES PREMIÈRES CONCESSIONS À SILLERY PAR MONTMAGNY}

Le père Campeau s'est très bien exprimé sur l'importance de la Grande-Allée (ou «Grande Route de Kebec au Cap Rouge»), cett= ligne tracée par l'ingénieur Bourdon dès avant 1640 et qui, partant du fort Saint-Louis va, traversant la censive des Cent-Associés en ligne droite, jusqu'à une demie lieue de la rivière du cap Rouge: «Elle est essentielle à l'organisation territoriale de toute cette superficie, non seulement de la banlieue, mais aussi de la seigneurie des Sauvages qui y fera suite ${ }^{10} . »$

8. Le 12 décembre 1720, l'arpenteur Noël Bonhomme-Beaupré procède au bornage de la route de la Grande-Allée, à Sillery; MRNQ, chemise S.3/8. En janvier 1754, Ignace Plamondon fait de même; MRNQ, chemise S.3/9.

9. Son tracé était rendu sensible, sur le terrain, par l'utilisation de signes convenus: branches cassées, arbres plaqués, pieux de cèdres plantés dans le sol ou bornes de pierres placées à certaines distances.

10. L. Campeau, loc. cit., 11. 
Et la première description que nous possédions d'une terre située sur le futur emplacement de la seigneurie de Sillery, et où il est fait mention du «chemin de Québec au Cap-Rouge», se trouve dans l'acte de la concession des cent trente arpents qui est accordée le 3 juin 1637, par le gouverneur Montmagny, à François Derré de Gand, commissaire général des Cent-Associés: «[...] avons distribué et départy cent arpens de bois ou environ au-dessus dudit costeau, bornez du costé du nord une ligne parallelle au chemin qui va de Québecq au Cap Rouge ${ }^{11}$.»

Nicolas Pelletier et Thomas Hayot, quant à eux, obtiendront du même gouverneur, les 12 septembre 1645 et 15 avril 1646, une terre de cinquante arpents en superficie bornée, l'une et l'autre, en des termes similaires:

[...] d'un bout au Sudest par une Ligne qui court sudouest et nordest esloignee du bord du grand fleuve st. Laurens de vingt toises environ; de L'autre bout, au nordest aussi par une ligne qui court sudest et nordest, ou environ, Laquelle ligne est eloignee du grand chemin de Kebec au Cap Rouge de douze toises $[\ldots]^{12}$.

Dans ces actes de concession délivrés à Derré de Gand, Hayot et Pelletier, et dont nous n'avons reproduit qu'une partie, il n'est question que de lignes, parallèles ou perpendiculaires au «grand chemin de Kebec au Cap Rouge», mais de lignes seulement, qui permettent le bornage de ces nouvelles concessions.

Vient ensuite, le $1^{\text {er }}$ août 1646 , en faveur des Mères hospitalières, une concession de cent trente-six arpents, en deux portions dont l'une est délimitée de la façon suivante:

[...] Scavoir d'un coste au Nortest en partie le ruisseau saint Andre, Et en partie par une route qui court au nortouest ou environ qui est perpendiculaire au chemin qui va de Quebec au Cap Rouge, Dautre coste au Sud-ouest par une route qui court aussy au nort ouest Et qui est perpendiculaire audit Chemin qui va de quebec au Cap rouge $[\ldots]^{13}$.

Dans ce passage, le mot «ligne»a été remplacé par «route» en conservant, néanmoins, le sens du précédent: il s'agit toujours d'une délimitation, d'un repère d'arpentage. Comme dans cet extrait de

11. Le texte de cette concession se trouve reproduit dans l'acte de ratification qui en a été donné par les Cent-Associés, le 5 avril 1639; L. Campeau, Monumenta Novae Franciae (IV), document 71: 204-208. Bien entendu, nous ne possédons pas cette carte qui nous serait, aujourd'hui, d'une grande utilité.

12. ANQQ, Fonds Biens des Jésuites, boîte E21/186.

13. Ibid. 
1650: «[...] toutes lesquelles routes ou lignes [...] serviront de bornes ou limites iusqu'a ce qu'il y soit planté des pierres avec brique et charbon, pour y servir de bornes en la manière accoustumee $[\ldots]^{14} . »$

On aura noté, dans ces deux derniers passages, que ce sont les termes «ligne» et «route» qui sont employés l'un pour l'autre et non pas «route» et «chemin».

Déjà depuis 1645 au moins, on avait pris l'habitude de préciser un usage additionnel à ces «lignes» ou «routes». Par exemple, les actes de concession à Pelletier et Hayot portent l'indication suivante: «lesquelles lignes serviront de chemin», et l'acte de concession aux hospitalières se termine sur des termes qui ne sauraient être plus clairs: «Lesquelles routtes sont de Dix huit pieds de large qui serviront de Chemins».

Il faut voir, dans cette indication de la part de Montmagny, la preuve supplémentaire qu'une route, dans l'emploi qu'on en fait alors, n'est pas un chemin sans quoi le gouverneur n'aurait pas jugé nécessaire de préciser sa double fonction. Si ces termes avaient été synonymes, il lui aurait suffi de dire : «Lesquelles routtes sont de Dix huit pieds de large». C'est donc qu'à sa première utilité, aux fins de bornage et d'arpentage, on a voulu lui en adjoindre une autre, celle de chemin praticable, qu'il fallait alors préciser.

Enfin, le 2 août 1646, le même gouverneur concède aux Amérindiens de Sillery une terre sur la rive nord du Saint-Laurent, en ces termes: «[...] sçavoir d'un costé la route de Puiseaux, d'autre costé un ruisseau qui les sépare d'avec celles des révérends Pères de la Compagnie de Jésus, d'un bout la grand route qui va de Québecq au Cap Rouge, d'autre bout le fleuve Sainct-Laurent $[\ldots]^{15} . »$

Dans ce dernier passage on aura noté l'emploi des expressions «route de Puiseaux» et «grand route qui va de Québecq au Cap Rouge». Si la première expression s'applique à la ligne perpendiculaire au fleuve, qui marque la limite sud-ouest de la terre du sieur de Puiseaux, l'autre renvoie à cette ligne que l'ingénieur Bourdon a jadis tracée d'un bout à l'autre de la banlieue et qui devrait se confondre avec le chemin du même nom. Alors comment expliquer l'emploi de ce terme d'arpentage de préférence à celui d'une réalité bien plus tangible, le chemin praticable, déjà évoqué dans les actes S.3/2.

14. MRNQ, «Procès verbal des 336 arpans du Sieur dAuteuil, 9 mars 1650»; chemise

15. L. Campeau, $M N F(\mathrm{VI})$, document 111: 487-488. Le texte de cette concession comporte une difficulté: cette terre des Amérindiens ne peut être bornée au sud par le fleuve Saint-Laurent, puisque l'anse de Sillery, appartenant maintenant aux Jésuites, s'interpose entre les deux. Montmagny semble l'avoir oublié lors de la rédaction de cet acte. 
précédents. La raison en est simple: au moment où Montmagny s'exprimait de la sorte, le «chemin de Québec au Cap Rouge» n'existait pas encore!

C'est ce que nous rappelle Bourdon, dans un procès-verbal d'arpentage et de bornage du fief de Monceaux, rédigé le 15 mars 1650, et qui s'exprimant au sujet du chemin de Québec au Cap Rouge qui devait servir de borne à la concession de 1646, le fait en ces termes: «d'autant que le grand chemin qui doict aller de Kébec au cap Rouge n'est encore fait et n'ayant la cognoissance directe par où il doibt passer, et ayant esté pris pour borne imaginaire de la concession des 120 arpans ${ }^{16} . \gg$

Le «chemin de Québec au Cap-Rouge», malgré le fait que l'on en ait jadis parlé comme d'une chose réalisée, tarde donc à être construit. On peut le comprendre aisément: avant l'arrivée à Sillery et à Gaudarville de colons en nombre suffisant, on ne voit pas qui aurait pu s'y employer. Et lorsque ce chemin sera enfin réalisé, son parcours, dans sa majeure partie, n'empruntera pas le même tracé que celui de la ligne ou route de la Grande-Allée. Il passera à quelque distance au sud de celle-ci. Son parcours ne sera pas rectiligne mais plus ou moins parallèle au bord de la falaise, à une distance variant, d'est en ouest, de 16 à 12 arpents.

\section{LES ROUTES DE SILLERY NE SONT PAS DES CHEMINS}

Si le chemin de Québec au Cap-Rouge n'a pu se réaliser entièrement selon le tracé de la route du même nom, en est-il de même pour les autres routes de Sillery? Nous croyons que oui. Et nous voulons tirer argument du fait de l'existence d'une exception, celle de la route ou ligne nord-est de bornage du fief de Monceaux, perpendiculaire à la Grande-Allée. En effet, cette route devint un véritable chemin de traverse reliant en ligne droite le chemin Sainte-Foix, où était située la deuxième église de Sillery (v1673-1698), à l'anse SaintJoseph, au bas de la falaise, lieu de résidence des pères jésuites. Et même si ce chemin fut délaissé, sans doute peu de temps après que l'emplacement de la troisième église de Sillery fut choisi, beaucoup plus à l'ouest sur le chemin de Sainte-Foy, nous en conservons la trace dans des documents postérieurs: par exemple, si, en 1682, la terre de Charles Maufay est bornée d'un côté par «le grand chemin qui va de St Michel à Sillery», cette même terre, en 1717, est dorénavant bornée par «la routte ou ancien chemin qui vat de la coste St Michel a Sillery».

16. MRNQ, «Procès verbal...»; chemise S.3/2. 
À notre sens, cette exception démontre clairement que si les routes de Sillery étaient devenues de véritables chemins nous en retrouverions la trace dans les documents de l'époque. Mais nous avons eu beau parcourir tous les actes de concession ou de vente, et surtout les procès-verbaux d'arpentage des terres contenues dans les quatre côtes, il n'est nulle part question - à part le seul exemple précédemment cité - d'anciens chemins, mais toujours de «routte» ou bien de «ligne ou routte». Nous croyons que cette absence constitue en soi la preuve que ces routes de Sillery, bien qu'imaginées par les Jésuites, selon un plan logique d'aménagement territorial de la seigneurie, ne devinrent jamais des chemins. En fait, elles ne remplirent qu'une seule de leur double fonction possible: délimiter le front de certaines côtes (SaintIgnace, Saint-Michel et Saint-Pierre) et la profondeur d'une autre (Saint-François) ${ }^{17}$. Et c'est dans cet esprit que la carte de Plamondon, de 1754 , en conserve le souvenir.

\section{LEXICOLOGIE FRANÇAISE DU XVII SIÈCLE}

Nous ne saurions clore l'examen de cette question sans avoir jeté un coup d'œil dans le Dictionnaire de Furetière, afin de vérifier si tout ce qui semble se dégager des documents d'époque et de notre hypothèse concernant l'emploi du mot «route» trouve un écho favorable dans ce recueil lexicographique de la langue française, rédigé à la fin du XVII ${ }^{\mathrm{e}}$ siècle ${ }^{18}$.

Commençons avec «chemin», le moins ambigu des deux termes pour désigner une voie praticable. Furetière le définit ainsi: «Passage qui est au public pour aller d'un lieu à un autre [...] Les grands chemins, ou proprement chemins de charroy, selon la Coûtume de Clermont, sont des chemins de trente pieds de large; \& les chemins royaux en ont soixante-quatre, \& dans les forests soixante ${ }^{19}$.»

Quant aux quelque neuf acceptions différentes pour le mot «route», trois ont attiré plus particulièrement notre attention. Tout d'abord, la première, l'acception la plus usuelle: "Chemin public connu \& fréquenté pour aller d'une ville ou d'une Province à l'autre ${ }^{20} . »$ Au Canada, c'est notre «chemin du Roy» qui assure cette fonction.

17. Nous disons «double fonction possible», car, en fait, il n'est pas certain que les Jésuites aient souhaité que les «routes» de Sillery deviennent des chemins.

18. A. Furetière, Le dictionnaire universel (Paris, SNL-Le Robert, 1978), 3 tomes. Cette édition moderne est la reproduction de l'édition de 1690.

19. Ibid., tome I.

20. Ibid, tome III. 
Mais ce qu'il y a de très intéressant pour notre propos, ce sont les deux acceptions suivantes. Premièrement: «se dit aussi des grandes allées qu'on coupe dans un parc, dans une forest, tant pour l'ornement, que pour la commodité de la chasse \& le passage des Voyageurs». C'est ni plus ni moins notre «grande route de Kébek au Cap Rouge dite la Grande Allée» qui fut tracée par Bourdon et qui traverse de part en part la banlieue de Québec, à l'époque de sa plus grande expansion.

Deuxièmement: «en termes de Marine, est le sillage ou le rumb de vent sur lequel il faut naviguer pour arriver à un certain lieu, \& que le Pilote suit en observant toujours la boussole». Voilà, à notre avis, l'origine du terme «route», employé de façon si caractérisée sous la plume de Montmagny et des premiers arpenteurs de la région de Québec. En effet, dans un procès-verbal d'arpentage des terres de la seigneurie de Gaudarville, il est précisé que l'arpenteur Jean GuyonDubuisson «a posé la boussole tiré une ligne perpendiculaire suivant nord-est sud-ouest 4 degrés ${ }^{21}{ }_{\gg}$. Et dans cet autre procès-verbal d'arpentage de la «ligne de la grande allée», l'arpenteur Noël BonhommeBeaupré précise: «la ditte ligne allant sud ouest quatre degrez trois quarts environ vers le nord ouest ma boussole declinant de quinze degrez ${ }^{22}$.» $\mathrm{Ce}$ dernier emploi serait donc tiré du vocabulaire de la marine en raison de la similitude du moyen employé, la boussole, qui seule permet de tirer cette ligne d'arpentage et de bornage qu'est la «route».

Comme on vient de le voir, les différentes acceptions du terme «route», en usage au XVII ${ }^{\mathrm{e}}$ siècle, laissaient un certain nombre de choix parmi lesquels nos ancêtres n'eurent qu'à choisir au détriment de son acception la plus courante.

\section{ROUTES OU CÔTES}

Nous allons maintenant tenter de démontrer que, dès 1670 , le terme «route» a connu, en plus de son emploi usuel, un glissement de sens. Ayant servi à désigner à l'origine une simple ligne d'arpentage, le terme sera utilisé - dans un nombre limité de cas, certes, mais sera utilisé tout de même - pour désigner la côte entière avec laquelle elle partage le nom.

21. MRNQ, 13 octobre 1667, arpentage et bornage des terres du Cap-Rouge; chemise S.3/4. Par ailleurs, il est intéressant de noter que: «en termes d'Architecture, d'Arpentage et de Jardinage, on appelle ligne, le cordeau avec lequel on trace sur terre les desseins des bastiments, on mesure les longueurs, on dresse les allées»; Furetière, op. cit. On voit ici que l'instrument a fini par prendre le nom de la figure géométrique qu'il permet de tracer ou de mesurer.

22. MRNQ, «Route de quebec au cap rouge ou grande allé, 12 décembre 1720 , Noël Beaupré»; chemise S.3/8. 


\section{TABLEAU 1}

Répartition des actes selon les gouvernements et les mentions de génériques de lieux contenus dans les sommaires de Parchemin

\begin{tabular}{||l||c|c|c||}
\hline \multicolumn{1}{||}{} & QUÉBEC & TROIS-RIVIÈRES & MONTRÉAL \\
\cline { 2 - 4 } GÉNÉRIQUES & 89208 & 17141 & 104031 \\
\hline \hline paroisse & 32252 & 1434 & 14885 \\
\hline seigneurie & 27165 & 5465 & 17109 \\
\hline côte & 10329 & 316 & 16593 \\
\hline route & 597 & 6 & 1 \\
\hline chemin & 251 & 31 & 116 \\
\hline
\end{tabular}

TABLEAU 2

Correspondance sémantique entre les doublets «route/côte» et «route/chemin»

\begin{tabular}{||l|c|}
\hline \multicolumn{1}{|c|}{ SÉQUENCES } & ACTES \\
\hline \hline «route ou côte...» & 50 \\
\hline «route ou chemin...» & 23 \\
\hline «chemin ou côte...» & 0 \\
\hline
\end{tabular}


Pour ce faire nous avons eu recours aux sommaires d'actes de la banque de données notariales $\operatorname{Parchemin}^{23}$. Celle-ci offre, pour chacun des 210380 actes notariés, rédigés sous le Régime français et conservés jusqu'à nos jours, un bref résumé variant de une à une vingtaine de lignes. Il faut bien comprendre qu'un pareil travail n'a été possible que dans la mesure où l'on a retenu, lors de la saisie des données, que les paramètres les plus importants de l'acte. Aux fins de notre étude, nous ne retiendrons que le paramètre qui concerne les mentions de lieu de résidence des individus et les mentions de lieu d'emplacement des terres quand celles-ci font l'objet d'une transaction.

Les toponymes - ou spécifiques - se rencontrent dans les actes, accompagnés ou non de leur générique: «seigneurie de Sillery» ou «Sillery», par exemple. Seuls les génériques retiendront notre attention: «seigneurie», «paroisse», «côte» et «route». Comme on peut le constater dans le tableau 1, le générique «côte» qui, associé à un spécifique, sert à préciser, à l'intérieur d'une seigneurie ou d'une paroisse, le lieu de résidence d'un individu ou celui de l'emplacement d'une terre, est d'un usage relatif si on le compare à "paroisse» ou à «seigneurie»: ce terme n'apparaît que dans $9 \%$ seulement des sommaires d'actes.

Dans un premier temps, nous avons cherché à établir, en comparant les fréquences de l'emploi des séquences «route ou côte», «route ou chemin« et «côte ou chemin«, si ces trois génériques étaient interchangeables entre eux. Du tableau 2, il ressort clairement que si le générique «route» peut être associé à celui de «chemin» et de «côte», ces deux derniers, pour leur part, ne le sont jamais entre eux. Dans le premier cas, on a vu plus haut de quelle manière le terme «route» a pu être associé à celui de «chemin»: le tracé d'au moins deux routes (ou lignes d'arpentage) ont effectivement servi de support aux chemins qui se confondent alors avec elles ${ }^{24}$. Quant à la séquence «route ou côte», elle ne laisse aucun doute sur l'équivalence séman-

23. On ne saurait remettre en question la rigueur de cette méthode du seul fait qu'elle s'appuie sur les sommaires d'actes qu'en a dressés la banque de données notariales Parchemin. C'est ainsi que pour avoir reconstitué le terrier de Sillery pour la période 1652-1723 (et dans certains cas jusqu'en 1754), nous pouvons affirmer, copies des originaux en mains, que les mentions de résidence des individus ainsi que les mentions d'emplacement des terres correspondent exactement aux informations contenues dans les sommaires de Parchemin: un coup de sonde, à travers notre corpus comportant une cinquantaine d'actes, nous a permis d'arriver à cette certitude.

24. C'est le cas notamment de la Grande-Allée: route et chemin se confondent jusqu'à un certain moment dans la banlieue, après quoi ce dernier bifurque vers le sud, tandis que la route du même nom poursuit son tracé rectiligne jusqu'à la limite sud-ouest de Sillery, et ce, durant toute la période du Régime français, ce qu'atteste la carte de Plamondon (1754). C'est le cas aussi de la route ou chemin de Saint-Claude, à Notre-Dame-des-Anges; voir tableau 3. 
tique entre ces deux termes. $\mathrm{Si}$, dans 50 actes, des individus ont pu se dire «de la route ou côte de», ils ne se sont cependant jamais dits «du chemin ou côte de». Ce qui nous paraît concluant en soi ${ }^{25}$. Enfin, comme si cela ne suffisait pas à emporter l'adhésion, rappelons ce passage d'un document autographe de juin 1718, où le père Raffeix, procureur des Jésuites, décrit la seigneurie de Sillery comme étant «partagée en quatre contrées ou routes ${ }^{26}$ ».

\section{UN RÉGIONALISME}

Examinons maintenant les tableaux 3 et 4 . Il apparaît de façon surprenante que l'emploi du terme «route», dans le sens de celui de «côte», constitue un régionalisme sur toute la période du Régime français. Il ne se rencontre que dans le Gouvernement de Québec et,

\section{TABLEAU 3}

Répartition des actes de 24 notaires, provenant du Gouvernement de Québec, sous le Régime français, contenant le terme «route» dans les mentions de résidence d'individus ou d'emplacement de leur terre

\begin{tabular}{|c||c|c||}
\hline PÉRIODE & RÉSIDENCE & EMPLACEMENT \\
\hline \hline $1650-1669$ & 0 & 11 \\
\hline $1670-1689$ & 64 & 96 \\
\hline $1690-1709$ & 23 & 68 \\
\hline $1710-1729$ & 1 & 107 \\
\hline $1730-1749$ & 1 & 59 \\
\hline $1750-1765$ & 2 & 57 \\
\hline \hline TOTAL & 91 & 398 \\
\hline
\end{tabular}

25. Et puis, certains libellés d'actes ne laissent place à aucun doute possible; par exemple, cette concession faite à Guillaume Deguise, dans la côte Saint-Pierre: «deux arpents de terre de front situez en la dite route St Pierre sur la riviere Saint Charles, et de profondeur depuis la dite riviere jusqu'a la ligne qui separe la dite route St Pierre d'avec celle de St Michel»; ANQQ, Genaple, 17 décembre 1703. Dans cet exemple, l'expression «route St-Pierre» ne désigne plus une ligne d'arpentage: il est nécessaire de remplacer «route» par «côte» pour que cet acte soit intelligible.

26. ANQQ, «Quelques Remarques sur la carte de la Seigneurie de Silleri, par le p. Raffeix»; boîte E21/189. 
TABLEAU 4

Répartition des actes contenant le terme «route» ou la séquence «route ou chemin», dans la banlieue et les seigneuries

\begin{tabular}{||l||c|c||}
\hline \multicolumn{1}{||c||}{ BANLIEUE } & ROUTE/CÔTE & ROUTE/CHEMIN \\
\cline { 2 - 3 } & \multicolumn{2}{c||}{} \\
\hline \hline Grande-Allée & 21 & 5 \\
\hline Saint-Jean & 8 & 0 \\
\hline \hline \multicolumn{1}{||c|}{ SEIGNEURIES } & \multicolumn{2}{||}{} \\
\hline \hline Notre-Dame-des-Anges & 34 & 18 \\
\hline Saint-Ignace & 86 & 0 \\
\hline Saint-Gabriel & 190 & 0 \\
\hline Sillery & 140 & 0 \\
\hline Gaudarville & 14 & 0 \\
\hline Maur (de) & 29 & 0 \\
\hline Neuville & 7 & 0 \\
\hline \hline TOTAL & 529 & 23 \\
\hline
\end{tabular}

à l'intérieur de celui-ci, dans quelques seigneuries seulement: de façon notable, à Saint-Gabriel, Sillery, Saint-Ignace et Notre-Dame-desAnges et, dans une moindre mesure, à de Maur, Gaudarville et Neuville.

Comment expliquer un tel phénomène? Tout d'abord, il faut faire la distinction entre l'emploi du terme «route», comme ligne d'arpentage, et l'autre, comme côte, né d'un glissement de sens. En effet, si le premier a pu être introduit ailleurs que dans les seigneuries énumérées plus haut - ce dont les sommaires de Parchemin ne saurait rendre compte - , le deuxième n'apparaît que sur un territoire bien circonscrit.

Considérons les seigneuries déjà établies, en 1663, dans le Gouvernement de Québec: elles sont au nombre de onze. À part Neuville, nos sept seigneuries en font partie, auxquelles il faut ajouter Beaupré, Beauport, Saint-Joseph, l'Île-d'Orléans et Lauzon. Ces deux 
dernières sont pourvues de routes d'arpentage: la première, qui la traverse de part en part en son milieu; la seconde, dans la profondeur de sa première côte riveraine ${ }^{27}$. En fait, à l'exception de Beaupré, Beauport et Saint-Joseph, toutes ces seigneuries portent l'empreinte de l'arpenteur Jean Bourdon ${ }^{28}$. Cette condition, nécessaire, n'est cependant pas suffisante dans l'apparition de ce régionalisme. Il faut chercher ailleurs l'explication: là où se trouve concentré l'emploi de la «route/côte». Et c'est à Saint-Gabriel, Sillery, Saint-Ignace et Notre-Dame-des-Anges: trois de ces seigneuries sont administrées par les Jésuites ${ }^{29}$. Nous identifions donc cette condition comme suffisante à l'apparition de ce régionalisme.

$$
\text { * * }
$$

On a déjà qualifié le mode de concession sous forme de côtes, en Nouvelle-France, de «système neuf, géométrique (très XVII siècle $)^{30} »$. Et en ce sens, comme Lucien Campeau, nous serions enclins à croire que l'influence des Jésuites dans les mille et une modalités d'établissement de la colonie française en Amérique fut déterminante. Leur cosmopolitisme ainsi que leur haut degré d'instruction leur conféraient une autorité dont les premiers administrateurs de la colonie ne purent que vraisemblablement profiter. Il n'est pas inutile de rappeler, à ce propos, que Mary Ann La Fleur et Marcel Trudel ont déjà attiré l'attention sur l'erreur habituelle qui consiste à attribuer la paternité du modèle en étoile des villages de «Bourg Royal» et de «Charlesbourg» à Jean Talon plutôt qu'aux Jésuites, ses véritables concepteurs ${ }^{31}$.

27. C'est le cas notamment à l'île-d'Orléans et à Lauzon, comme l'a noté Marcel Trudel; op. cit., 55 et 485 .

28. Beauport est un cas particulier: la profondeur de la première côte n'est pas parallèle au Saint-Laurent, mais se confond avec la limite nord-est de la seigneurie; Trudel, op. cit., carte 13. Saint-Joseph est établie dès avant l'arrivée de Bourdon en Nouvelle-France et les terres concédées par la suite sont de profondeur variable, ce qui exclue une ligne droite d'arpentage. Enfin, Beaupré, propriété du Séminaire de Québec, nous laisse perplexe: deux routes d'arpentage y apparaissent mais tardivement, en 1752 !

29. Il n'est pas impossible que les Jésuites aient secondé les hospitalières, propriétaires de Saint-Ignace, contiguë à Saint-Gabriel, dans l'administration de leur fief. Quant à Gaudarville, de Maur et Neuville (propriété de l'arpenteur Jean Bourdon), comme ces seigneuries font géographiquement suite à Sillery, ne pourrait-on pas parler, dans ces cas, d'un effet par contamination?

30. P. Deffontaines, «Le rang, type de peuplement rural du Canada français», Cahiers de Géographie, 5 (1953): 29.

31. Mary Ann La Fleur, «Community Development in Seventeenth-Century New-France: Notre Dame des Anges», P. Benes et J. M. Benes, eds., New-England/New France 1600-1850 (Boston University, 1992). (The Dublin Seminar for New England Folklife. Annual Proceedings. 15 and 16 July 1989), 14: 60-68; Marcel Trudel, RHAF, 44,3 (hiver 1991). 
Au début de la colonie, cette conception géométrique de l'aménagement territorial a très bien pu trouver un accueil favorable chez Montmagny, ancien élève des Jésuites, ou chez les arpenteurs Jean Bourdon et Martin Boutet, autres familiers des disciples de Loyola. Rappelons que ce dernier, qui «enseignait les mathématiques, particulièrement dans leur application à l'arpentage et à la navigation», illustre bien le pont qui existait déjà entre ces trois disciplines ${ }^{32}$.

\section{CONCLUSION}

Une première conclusion s'impose: le «réseau routier» de Sillery ne doit pas être confondu avec son réseau de chemins ou voies praticables. Ce dernier emprunte un tout autre tracé.

Y a-t-il lieu, d'autre part, d'étendre cette conclusion à toutes les seigneuries où le terme de «route» eut cours? Nous pensons pouvoir répondre par l'affirmative. Les cartes cadastrales de 1754, levées par Plamondon, pour les seigneuries de Sillery, Saint-Gabriel et NotreDame-des-Anges nous invitent à conclure en ce sens.

Quant à l'emploi du régionalisme «route/côte», limité à quelques fiefs, mais cependant utilisé tout au long du Régime français, il perpétue, croyons-nous, à travers des réflexes quotidiens, tant de la part des Jésuites que de celle de leurs censitaires, le souvenir d'une époque où l'aménagement territorial était conçu selon un plan ordonné, géométrique, au mépris des contraintes d'ordre géographique.

32. «Boutet de St-Martin, Martin», $D B C, \mathrm{I}: 122$. 